\title{
L'institution fait son cinéma. Retour sur l'exposition Nouvelles vagues au Palais de Tokyo
}

\section{Nicolas Boutan}

\section{(2) OpenEdition}

1 Journals

Édition électronique

URL : http://journals.openedition.org/communicationorganisation/4739

DOI : 10.4000/communicationorganisation.4739

ISBN : 979-10-300-0155-6

ISSN : 1775-3546

Éditeur

Presses universitaires de Bordeaux

Édition imprimée

Date de publication : 1 décembre 2014

Pagination : 85-98

ISBN : 978-2-86781-905-6

ISSN : $1168-5549$

\section{Référence électronique}

Nicolas Boutan, "L'institution fait son cinéma. Retour sur l'exposition Nouvelles vagues au Palais de Tokyo », Communication et organisation [En ligne], 46 | 2014, mis en ligne le 01 décembre 2015, consulté le 26 avril 2019. URL : http://journals.openedition.org/communicationorganisation/4739 DOI : 10.4000/communicationorganisation.4739 


\title{
L'institution fait son cinéma Retour sur l'exposition Nouvelles vagues au Palais de Tokyo
}

\author{
Nicolas Boutan ${ }^{1}$
}

Interrogé par Bernard Pivot à l'issue de sa mandature sur le bilan qu'il dressait de la campagne des "grands travaux ", le président François Mitterrand illustra sa réponse avec l'anecdote suivante : «Jadis, cheminant dans Lutèce et croisant un groupe d'ouvriers occupés à poser pierre sur pierre, un touriste leur demanda ce qu'ils étaient en train de faire. Ils répondirent qu'ils empilaient des pierres les unes sur les autres. Peu après, le touriste rencontra un second groupe visiblement occupé à faire la même chose. Or, à la même question ils répondirent qu'ils étaient en train de construire une cathédrale ». Cette historiette accrédite l'épure du projet proposée par Jean-Pierre Boutinet :

Tout se passe comme si les activités à projet impliquaient une dynamique à deux temps : le premier temps est consacré à l'esquisse obligatoire de l'activité à réaliser, qui fera fonction de schéma directeur, ce que l'italien appelle progetto. Une telle esquisse vise à anticiper le second temps, qui est la réalisation de l'activité proprement dite, «la progettazione» des Italiens. (Boutinet, 2011 : 99)

Est-il permis d'interpréter à travers la réponse présidentielle une volonté non dissimulée de distinguer entre la trivialité d'une action pour l'action de celle qui s'inscrit dans un grand dessein - la « vision » de l'auguste homme c'est-à-dire de celui capable de lire les augures, de les prévoir ? Partant de cette approche du projet, où situer le design ? Comme optimum, le design permet de mesurer l'écart ou le pas de côté des premières intentions (dessein). Du point de vue de la conception et des «sciences de l'artificiel » décrites par Herbert Simon, le design nous semble procéder d'une pré-occupation, d'un souci, principal mobile de ce que Donald Schön a qualifié de « pratique réflexive » et que l'on pourrait étendre jusqu'à " pratique soucieuse ». C'est à partir de cette perspective du souci que nous voudrions proposer l'observation

1 Nicolas Boutan est chargé de cours en arts et design à l'université Bordeaux Montaigne et doctorant au sein du Laboratoire MICA - EA44 26 où il conduit une thèse intitulée Quand le design crée l'événement esthétique du curating; nicolas.boutan@u-bordeaux3.fr 
d'une pratique singulière relevant du projet, la curation. Popularisée à l'ère numérique, la curation consiste à sélectionner, trier, agencer des données disponibles (les data) sur le web à des fins d'organisation et de présentation. Du point de vue de l'art contemporain, la curation (ou curating) désigne également les agencements d'œuvres orchestrés par les curateurs dans les expositions : "C'est le défi qui est lancé à tous ceux qui sont maintenant immergés dans une culture massivement imagée : construire le langage et les codes d'organisation de ces flux. » (Menger, 2005 : 39). Plutôt que le terme curation et afin d'insister sur le soin apporté à la mise en espace de ces œuvres, on évoque la formule pratique curatoriale, devenue symptomatique d'un nouveau régime de l'administration de l'art contemporain face à l'accumulation des expériences esthétiques: «Aujourd'hui, au-delà de l'analyse et de l'enrichissement d'une collection particulière s'affirme le besoin d'une relecture générale de l'histoire de l'art moderne et contemporain » (Grenier, 2013 : 38). Nous avons choisi de retenir le terme curator car il évoque le souci qui accompagne l'action de ces protagonistes de la scène contemporaine plutôt que le statut de délégué auquel renvoie « commissaire». En orchestrant des navigations possibles à travers des données en perpétuelle augmentation, le phénomène du curating s'impose au cœur de l'institution culturelle (musée, centre d'art ou bien Fonds régional d'art contemporain) et favorise une mise en tension projet-design. En effet, devant la masse d'informations, de manifestations et d'événements concurrents, les assemblages d'œuvres que les curators construisent guident les publics à travers des pistes de réflexion, des points de vue, des positionnements. Par conséquent, cette activité conduit à s'interroger sur la nature de l'accès aux données, leur mise en forme, leur design. Sur les discours qui l'accompagnent, aussi. Si ce souci semble participer à la quête permanente de la solution la meilleure en fonction des contraintes, des acteurs, des demandes et du contexte, il reste encore à décrire et à observer à partir de la description qui en est faite par les acteurs de l'institution qui accueille l'exposition.

Pour ces raisons, une approche de la conduite de projet d'exposition temporaire d'art contemporain à partir de la représentation qui en est faite par l'institution nous a semblé présenter quelque pertinence. Car si cette pratique est assurée par des curateurs ou curators, souvent confondus dans un grand amalgame avec les conservateurs nationaux du patrimoine, les assistants de conservation, les scénographes et les commissaires d'exposition, elle alimente plusieurs discours sur différents supports : catalogue, aide à la visite, affiches, médiation orale, informations sur le site, articles de presse, etc. Ces discours favorisent à leur tour une mise en représentation qui en modélise et dessine les contours. Par exemple, un cas récent d'exposition tiré de l'actualité nous apparaît éloquent pour témoigner de cette modélisation d'un espace singulier du projet. Il s'agit, dans le cadre de la biennale de Venise en 2013, du remake de l'exposition célèbre Quand les attitudes deviennent forme, inauguralement 
présentée à Berne en 1969 par Harald Szeemann. Consacrée par la postérité comme le point de départ d'une position assumée de curateur (affranchie des artistes mais voisinant avec eux), son remake à Venise ajoute une dimension commémorative. De plus, le format du remake n'est pas sans évoquer les enjeux attachés à la pratique curatoriale et liés à la réécriture, à la citation, à la traduction : "Le style simple, clair, direct du re-writing vise à rendre le message transparent, à lui conférer une intelligibilité immédiate " (Morin, $2008: 48)$.

Nous avons voulu observer cette mise en représentation de la pratique curatoriale à partir d'un autre $\operatorname{cas}^{2}$ contemporain, l'exposition collective et temporaire Nouvelles vagues au Palais de Tokyo - Site de création contemporaine à Paris, qui a eu lieu durant l'été 2013 (21 juin - 8 septembre) 3 . Il s'agira d'envisager à travers cette analyse le rôle incontournable du projet de l'institution dans la diffusion d'une représentation des acteurs de la pratique curatoriale de l'art contemporain : "Si le cadre et le socle symbolisent cette structure auratique ajoutée, c'est la structure muséale tout entière qui, par la scénarisation de l'espace et l'inscription de l'objet d'art dans un parcours de visite, contribuent à sa valorisation et lui permettent de signifier en tant que tel » (Beyaert-Geslin, 2012:66).

Cette exposition associait vingt et une expositions dans les espaces de l'institution à quelque trente-deux autres manifestations dans des galeries d'art parisiennes dont le comité professionnel était partenaire de l'événement. La particularité de ce dernier consistait à exposer des projets curatoriaux et par voie de conséquence, des œuvres d'artistes. Habituellement, le rapport est inversé : c'est à partir de l'exposition d'œuvres d'artistes qu'un parti-pris curatorial peut apparaître en filigrane. Cette vaste exposition de plusieurs autres expositions invitait à deviner un préfixe adéquat : sur $/ \mathrm{méta} / \mathrm{para} / \mathrm{archi} /$ super-exposition. Les agents de l'institution affirmaient la volonté de « rendre manifeste l'émergence de la figure du curateur » (De Loisy, 2013 : 5). Pour cela, après qu'un appel à projets ait été lancé, un jury de personnalités (curateur/ rice(s) et directeur/rice(s) d'institutions) a examiné puis sélectionné parmi les candidatures celles qui seraient retenues. La pratique des curators s'impose désormais comme intention autonome à même de servir les intentions des artistes en assumant la responsabilité d'exposer leurs œuvres au mieux. Nous proposons dans le cadre de cet article de souligner le portrait du protagoniste de la construction d'exposition en designer « projeteur » (Vial, 2010) pour ouvrir le champ à une lecture des expositions sous l'angle du design à partir de la représentation en auteur-réalisateur de film suggérée par cette exposition au Palais de Tokyo.

Avant cela, il nous faut préciser le cadre de cette observation. En effet, les limites de l'étude d'une exposition d'œuvres d'art contemporain (quelles que

2 Le terme est employé ici au plus près de son étymologie qui renvoie à un accident, un accroc.

3 Cf. Catalogue de l'exposition : Jean de Loisy (dir.), Magazine Palais n 18, Paris, Ed. Palais de Tokyo, 2013. 
soient d'ailleurs les « formes » de ces œuvres) sont régulièrement ambigües, car comme le rappelle Yves Michaud : "Une exposition est un échantillonnage, une sélection ou un prélèvement : elle montre le plus représentatif, mais aussi ce qu'il a finalement été possible de montrer, c'est-à-dire de trouver, emprunter, déplacer ; bref, il faut faire de nécessité vertu " (Michaud, 1989: 135). Cette citation illustre combien les approches de l'exposition peuvent être aléatoires. S'en tiendra-t-on à sa propre expérience de visiteur ? Aux outils théoriques et méthodologiques de l'histoire de l'art ? De la sociologie ? De l'anthropologie ? Aux expositions de papier ? Une exposition de projets curatoriaux comme Nouvelles vagues invite à rechercher un ajustement, un assemblage des outils d'analyse à partir des discours disponibles : une expérience de visite commentée par un médiateur, le catalogue de l'exposition, des articles de presse, les informations disponibles sur le site du Palais de Tokyo. Même si l'art contemporain peut souvent être matière à discorde, il s'agira de montrer que sa mise en exposition emprunte au design et ouvre à la discussion : "Génératrice d'interactions critiques, cette pluridisciplinarité théorique place l'étude de l'art sous le signe de la discussion, ou encore de la dispute » (Grenier, $2013: 27$ ).

Quel était le " pitch » de l'exposition Nouvelles vagues ${ }^{4}$ ? Constituer un groupe de jeunes curateurs avant d'en organiser l'« émergence " représentative en singeant les cinéastes du mouvement de la Nouvelle vague en France. Le titre de l'exposition est suffisamment éloquent pour s'attarder sur les ramifications de sa signification. D'abord, sa formulation au pluriel qui abandonne l'unité d'un mouvement et lui préfère le déferlement ${ }^{5}$ de plusieurs nouvelles vagues. L'emploi du pluriel peut accréditer l'idée de l'impossibilité contemporaine à regrouper en un seul rassemblement tous ces projets qui empruntent des méthodes, des cultures de travail et des habitudes très variées. Si l'émergence de cette pratique est bien réelle, tout consensus à son sujet semble prématuré : diversité des statuts, des formations, des associations. Par le fait même, le renoncement à la globalisation de ces pratiques dans le cadre de l'exposition traduit aussi son caractère potentiellement incontrôlable ou créatif. Ce constat n'est pas sans renvoyer au mouvement de la nouvelle vague « version originale » : " Il n'y a, à proprement parler, ni mouvement, ni programme, ni école, ce qui provoquera toutes les ambigüités, confusions, malentendus. En outre, les cinéastes de la nouvelle vague ne demandaient qu'à s'insérer dans la production commerciale. Ce qu'ils ont réussi tôt ou tard, et on le leur a assez reproché ${ }^{6}$. La complexité du projet permet d'entrapercevoir combien les motivations d'exposition peuvent être variées, combinables, ajustables et

4 « En fait le pitch, par ce qu'on pense par pitch, c'est l'effet storytelling (...) », écrit Julien Fronsacq, « Le musée d'Art moderne de la Ville de Paris / ARC et le Palais de Tokyo : de quelques positions et collaborations inédites ». In Françoise Docquiert, Commissariat comparé. Histoires d'expositions, Paris, Paradox, 2009, p. 60. 5 Frédric Bonnet va jusqu'à parler d'un « vent frais curatorial », Le Journal des arts, 2013, n³95, juillet/ septembre, p. 10.

6 Notice Nouvelle vague, Encyclopédie Universalis. Disponible sur : www.universalis.fr 
combien elles sont devenues des territoires de création à part entière. On ne s'étonnera donc pas que l'exposition Nouvelles vagues établisse une référence explicite au monde du cinéma : "Dans la négociation complexe qui a lieu pour donner naissance à un film, il n'y a pas seulement, face à face, le studio et le producteur : dans le nouvel Hollywood, les interlocuteurs et les acteurs du système sont devenus innombrables » (Martel, 2010 : 97). Là encore, les projets d'exposition présentés illustrent une grande diversité d'approches selon les équipes qui les portent ou les accueillent.

Deuxième remarque, la nouvelle vague chasse la précédente et invite à considérer une rupture ${ }^{7}$, une opposition, avec la vague précédente. Seraitce la tabula rasa ? " (...) Nouvelle Vague en France, laquelle apparaît particulièrement emblématique de cette volonté de créer en dehors des standards de production, pour inventer un cinéma plus libre, reposant sur des structures moins lourdes, et donnant la priorité à l'auteur et à son travail de création sur la logique économique du producteur » (Lipovetsky et Serroy, 2013 : 203). On mesure toute l'ambivalence du choix d'une présentation associant différents projets curatoriaux à un mouvement cinématographique réclamant une "politique d'auteurs " dans l'un des lieux institutionnels de l'art contemporain parmi les plus importants en France. Nicolas Bourriaud, ancien directeur du Palais de Tokyo, a bien montré dans un ouvrage au titre éloquent, Potsproduction : la culture comme scénario. Comment l'art reprogramme le monde contemporain, les usages et pratiques communs à ces deux champs d'activités : réalisation de film et exposition. Mais ce rapprochement de la pratique curatoriale contemporaine au fonctionnement de la sphère du cinéma proche de l'industrie culturelle induit une menace commune, celle du mainstream telle qu'elle a été décrite par Fréderic Martel :

Aujourd'hui, dans le nouvel Hollywood, un film est financé par un studio qui le green light (le valide), mais qui ne le fait plus. Le produit est confié, sous le contrôle permanent d'agences de talent rémunérées au pourcentage sur toutes les transactions, à des milliers de sociétés indépendantes : des maisons de production, des start-up techniques, des PME spécialisées dans le casting, la post-production, les effets spéciaux ou la création de « trailers » promotionnels (Martel, 2010 : 97-98).

Cette description du verrouillage des produits dans le cadre de l'industrie cinématographique américaine explique que des opérations d'ajustement des structures de production et de diffusion soient programmées. Dans le cadre des institutions d'art contemporain, le constat d'une nouvelle adaptation s'affirme aussi. Catherine Grenier, conservateur au MNAM, décrit par exemple la nécessité pour le musée de développer une activité de production : "Pour s'adapter à l'évolution de la société, le musée devra devenir extensif,

7 Symptomatique, d'après Paul Ardenne, du monde de l'art contemporain : «La «rupture» est, dans le monde de l'art contemporain, une obligation formelle, un mot transitionnel, un impératif catégorique, sous cette condition institutionnelle : que rien, dans les faits, ne soit brisé. » Paul Ardenne, « Packaging first. L'exposant plus que l'exposé », Archistorm, été 2013, p. 126-131. 
flexible, permanent, et allier la fonction de conteneur à celle de producteur » (Grenier, 2013 : 83). La volonté des acteurs de l'institution qu'est le Palais de Tokyo d'opérer un rapprochement des projets de curateurs à un mouvement de réalisateurs de film exacerbe l'importance de l'économie de projet en place dans l'institution culturelle contemporaine centrée sur le curator : « Le réalisateur de cinéma, quant à lui, est un acteur clé dans une économie de projet et dans une organisation en équipe qui atteint couramment plusieurs dizaines voire plusieurs centaines de personnes » (Menger, 2005 : 82). Dans le cas de l'exposition Nouvelles vagues, il s'agit de convoquer ou de citer des réalisateurs d'un certain type : ceux qui ont réclamé à cor et à cri le statut d'auteur afin de représenter l'aspect créatif de leur travail. En effet « Les réalisateurs de la Nouvelle Vague se sont présentés comme des créateurs, des auteurs à la tête de petites équipes, en rompant avec le caractère assez routinier de la division du travail qui réservait au réalisateur une position de premier de cordée et de maître artisan " (Menger, 2005 : 82). À la suite de cette citation, l'on pourrait appliquer la « direction de petites équipes » d'artistes (et d'œuvres) aux curators qui les transportent dans leur sillage au cœur du Palais de Tokyo. De fait, cette exposition consacre les curateurs dont l'exposition est réalisée comme des porteurs de projet, des leaders, des chefs, ou bien encore des managers tels que brossés par Luc Boltanski et Eve Chiapello : "Ils deviennent donc animateurs d'équipe, catalyseurs, visionnaires, coachs, donneurs de souffle. » (Boltanski et Chiapello, 2011 : 130-131). Acteurs du projet de l'exposition dans lequel ils peuvent occuper plusieurs fonctions (conception, réalisation, diffusion, communication, ingénierie, évaluation, etc.), ils y puisent un caractère incontournable et nécessaire puisque c'est à eux que l'appel à projet était adressé. Curateur lui-même et parmi les plus connus (membre du jury de sélection de l'exposition), Hans Ulrich Obrist évoque (après Félix Fénéon) le terme « catalyst » pour définir le curateur.

Aussi le terrain de l'exposition collective temporaire (et thématique) Nouvelles vagues fournit-il une perspective à la relecture d'une importante tension de l'art actuel : le statut d'auteur du curator et la nature de ce qu'il crée, de ce qu'il catalyse. La rivalité artiste/curateur popularisée notamment par les propos de Daniel Buren à l'encontre d'Harald Szeemann ${ }^{8}$ a laissé la place à une cohabitation d'une nouvelle forme, pas nécessairement évacuée de tout recul critique, à l'image de la série photographique Be nice to your curator d'Erwin Wurm dans laquelle l'artiste se photographie en train de porter son conservateur à bout de bras. Il ne s'agit pas toutefois de nier les rapports de forces inhérents à un travail en équipe mais de les situer à une juste place sans les réduire à une équation binaire à deux éléments, quand

8 On pourra consulter l'entretien de l'artiste avec H.-F. Debailleux, «Les commissaires d'exposition ne doivent pas jouer aux auteurs ", Libération 21 juillet 2007, en ligne : http://www.liberation.fr/week-end/2007/07/21/ les-commissaires-d-exposition-ne-doivent-pas-jouer-aux-auteurs_98670 
la réalité est autrement complexe9. Dans un article au titre éloquent " Qui fait une exposition ? Du commissaire à l'équipe projet " (Scrive, 1994 : 99104), Martine Scrive revient sur son expérience à la Cité des Sciences et de l'industrie et décrit la diversité des parties prenantes, parmi lesquelles le curator et sa " bonification ", son regard.

Quelle est donc la nature de ce bonus (en sus) créé par le curator? Cette gratification, ce bénéfice que l'étymologie du terme bonus éclaire, serait nécessairement positive, lucrative. Dans ce cas, la pertinence du design pour mesurer ce supplément apporté au cœur de la conduite du projet d'exposition ne fait aucun doute. À la lumière des intentions premières du projet d'exposition jusqu'à l'étude des choix opérés dans la complexité, le design permettrait d'avancer des outils de lecture adaptés à une pratique curatoriale largement collaborative et contextuelle. En accueillant dans ses murs cette exposition à destination des curateurs, le centre d'art évoque son propre rôle, celui de catalyseur désormais émancipé de la constitution d'une collection à l'inverse du musée : « Le musée, par-delà son côté inventaire des formes, mais aussi précisément grâce à ce procédé, est un lieu de mémoire vive qui autorise des rapprochements toujours inédits entre les œuvres, un dialogue qui stimule l'imagination et agit sur l'artiste - qui continue à se rendre au musée - comme un révélateur et un catalyseur. » (Vander Gucht, 1998 : 35). Car c'est bien la sensation d'un tout unifié qui frappe lors de la visite dans les lieux : une progression au fil d'une ligne rose au sol qui conduit d'une exposition à une autre, seuls des adhésifs roses signalant un nouvel espace " autonome » accompagné de cartels mentionnant le nom du/ des commissaire(s), des artistes. En « piochant » dans le nombre des projets présentés quelques-uns des plus significatifs (visuellement, conceptuellement) dans leur discordance par exemple, la médiatrice opère un choix (préétabli par le service des publics) afin de tenir le cadre horaire de la visite (45 minutes). À l'identique d'une visite d'exposition dans laquelle les médiateurs ne sont pas en capacité horaire de présenter toutes les œuvres exposées, ici les projets se retrouvent liés comme des chapitres, des parties, des fragments d'un tout général qui est la surexposition de ces vingt et une expositions. C'est cette surexposition qui apparaît désignée, construite, scénarisée par l'institution et ses acteurs. L'interprétation par l'analyse des causes et conséquences des choix opérés, des réponses apportées aux problèmes rencontrés convoque de nombreux éléments, qui sont autant d'unités variables selon les contextes, les protocoles, les individus, les moyens, etc. Les choix opérés de concert entre le curator, les artistes et les acteurs de l'institution peuvent, dans le cas d'un projet d'exposition, porter à privilégier en vue d'objectifs précis, telle conduite plutôt

9 «Le projet est l'occasion et le prétexte de la connexion. Celui-ci rassemble temporairement des personnes très disparates, et se présente comme un bout de réseau fortement activé pendant une période relativement courte, mais qui permet de forger des liens plus durables qui seront ensuite mis en sommeil tout en restant disponibles. » (Boltanski et Chiapello, $2011: 170$ ). 
qu'une autre. Dans le cas de l'exposition Nouvelles vagues, la représentation de la pratique curatoriale en réalisation de films invite à la considérer comme le siège possible d'un manifeste théorique, d'une philosophie car elle est l'origine de la justification des choix opérés : « La théorie n'est, de fait, qu'un moment de la praxis : celui de l'appréciation des possibles " (Sartre, 1980 : 71). Les curators ayant pour charge l'appréciation des possibles en fonction des contraintes des expositions qu'ils présentent, c'est donc vers eux que se tournent les regards.

Cette revendication est importante et cruciale pour l'analyse de la situation contemporaine de la pratique curatoriale car le statut d'auteur réclamé par les réalisateurs de la nouvelle vague consiste à affirmer comme création la réécriture, avec style. Le style ${ }^{10}$, «la patte » d'un curateur. Autant de termes et de formules qui soulignent l'importance de la personnalité à l'origine d'une exposition qui s'incarne dans un projet -film ou exposition- car c'est elle qui justifiera le résultat d'une sélection, le fruit du « montage » sur lequel repose des attentes et motivations variées dont Yves Michaud se fait l'écho :

On attend d'abord d'une exposition un divertissement, que ce divertissement s'inscrive dans un comportement de loisir ou dans une démarche plus " utilitaire » d'éducation. Ce divertissement a bien des facettes, qui couvrent tout l'éventail de nos plaisirs visuels et au-delà : la stimulation de la curiosité, la quête de la fascination, l'exercice inhabituel de l'attention, un état d'absorption ou d'ingestion, la participation collective à un rite culturel, le renforcement de nos croyances en même temps que leur ébranlement. (Michaud, 1989: 139).

La conduite du projet d'exposition produit de la pensée. À travers la réception (visiteurs et pairs par exemple) et l'évaluation (institution par exemple), cette pensée suscite chez d'autres acteurs extérieurs (journalistes, critiques, artistes, agents, galeristes, collectionneurs) un motif de réflexion. De quelle nature est cette pensée ?

Le terme projet inclut un champ sémantique aussi large que général : c'est le moment préparatoire qui précède la réalisation d'un produit - qu'il soit matériel comme un objet ou immatériel comme un service - ou d'un support de communication, mais aussi tout cela en même temps. La mise en acte d'un projet est un procès stratégique finalisé : il s'agit d'un procès dynamique et changeant, composé de diverses étapes, de diverses figures professionnelles mais aussi de la convocation de diverses disciplines (Deni, 2009 : 91).

Si l'on applique cette définition du projet à l'exposition, alors c'est le design, pratique interdisciplinaire qui vient ici relancer la réflexion en proposant d'orienter et de guider ce bonus. Elle nous semble correspondre à deux axes

10 Cf. Morgan Jouvenet, «Le style du commissaire. Aperçus sur la construction des expositions d'art contemporain ", Sociétés E̋ Représentations 1/ 2001, n 11, p. 325-348 ; ou bien encore Jérôme Glicenstein "Qu'est-ce que le style d'un commissaire d'exposition? ». In Natacha Pugnet (dir.), Jeu(x) d'exposition. Pratique et théorie de l'exposition, Nîmes, École supérieure des Beaux-Arts, 2011, p. 122-135. (coll. Hôtel-Rivet). 
principaux : d'abord, la compréhension des situations, des choix opérés, mais parce qu'il faudra bien l'expliquer et la mettre en discours, la justifier, alors le curator doit argumenter et observer un recul sur ses choix d'où le constat d'un curator qui observe un recul sur ses actes à travers une pratique réflexive, collaborative (institution, artistes, prêteurs, prestataires). Les curators travaillent en fonction de contraintes établies (demandes d'autorisation de déplacements, assurances, itinérance des œuvres, délais d'ouverture d'expositions, obligations de dispositifs éducatifs annexes, etc.). De la même façon que le visionnage d'un film au cinéma peut être l'occasion de retrouver sur grand écran à travers les personnages des situations vécues ou fantasmées et de les vivre par procuration, par la médiation du film, le curateur peut projeter à partir de son scenario d'exposition des scènes (des dialogues ?) : "Rétrospectivement et prospectivement, nous projetons dans les œuvres nos préoccupations, intérêts, désirs et sentiments, lesquels ne se trouvent pas totalement en elles définitivement, ou bien nous y verrions toujours les mêmes problématiques » (Lageira, $2010: 42$ ). Si les œuvres « accueillent » ou occasionnent des projections diverses, c'est donc sur le design de ces projections que s'affairent les curators, en incarnant le projet, c'est-à-dire en lui prêtant chair. L'importance des personnalités ou de la personnalisation des signatures d'exposition n'est pas anecdotique. Bien au contraire, elle se retrouve parfois au cour des motivations de rentabilité des institutions culturelles. Le nombre d'entrées des expositions qui sera diffusé dans la presse, leur visibilité et leur rayonnement invitent à considérer comme un atout certain la signature de curators dont on devine qu'ils sauront proposer un « produit » attractif et conduire l'entreprise à bon port.

Indépendamment des critiques émises ici ou là sur ces produits formatés à destination du grand public qui ont pu conduire à une uniformisation et une réduction de l'offre muséographique locale et internationale, voire en certains cas à une baisse de qualité (le phénomène du " name dropping », basé sur le vedettariat), la politique des blockbusters pourrait atteindre très vite ses limites (Grenier, 2013 : 87).

Dans le même temps, le souci de visibilité et rayonnement accru depuis les années 2000 parmi les institutions culturelles fraye quelquefois avec des techniques de séduction commerciale empruntant à des sphères de l'industrie culturelle. C'est-à-dire l'exposition comme attraction, levier du désir, "drague " (teaser signifiant draguer est un mot américain utilisé pour les bandes annonces.) En cinéma, on parle aussi de trailer (pour bande annonce en français).

Du point de vue de l'exposition d'art contemporain, quel pourrait être le danger d'un tel basculement ? À considérer l'exposition comme un produit à lancer, il est probable que l'on en oublie l'œuvre en elle-même noyée dans un tout qu'il n'aura pas été possible de voir dans sa totalité ou dont il n'aura pas été possible de comprendre toute la démarche comme le remarque dès 2001 
Éric Mangion, directeur du FRAC PACA : «Les industriels - instruits pas les méthodes du marketing - savent mieux que quiconque qu'un produit se "vend" essentiellement avant sa sortie. La seule différence est qu'aujourd'hui la communication d'un produit fait souvent office de produit, et qu'il y a parfois plus d'intérêt dans un produit dérivé que dans un produit tout court » (Mangion, $2001: 35$ ).

De ce point de vue, la confession d'Anne Dressen, commissaire à l'ARC/ Musée d'art moderne de la ville de Paris, à propos de l'exposition de Rirkrit Tiravanija au couvent des Cordeliers en 2005 dans un entretien apparaît significative : «En fait, mon rôle a été finalement plus celui d'une directrice de casting » (Dressen, 2011: 54). La mission d'un directeur de casting consiste à recruter les acteurs, comédiens et figurants pour chacun des rôles à interpréter dans un film en fonction des contraintes de périodes de tournages, de booking des acteurs, des montants de leur cachet, du moment de leur carrière. Le curator peut prendre ce costume pour choisir les œuvres à exposer dans l'exposition et retenir, selon des contraintes similaires (lieu de conservation de l'œuvre, volonté de l'artiste ou du prêteur, frais d'assurance, conditions de transport, durée de visibilité, etc.). En proposant un bouquet de projets curatoriaux comme un nouveau mouvement d'auteurs, l'institution favorise un rapprochement au cinéma d'auteur qui laisse augurer pour le centre d'art le danger qui guette le cinéma d'auteur d'être concurrencé par le cinéma des blockbusters:

Ne trouve-t-on pas, à l'entrée des expositions et en ouverture des mégacatalogues, de véritables génériques où les artistes occupent la place des stars, où le commissaire assume la place du réalisateur; où il nomme et remercie ceux qui ont permis de produire la superproduction : sponsors, prêteurs, transporteurs, architectes des décors et bientôt grands couturiers ayant réalisé le costume non seulement des gardiens, mais des artistes eux-mêmes ? (Michaud, 1989b : 5).

Cette citation d'Yves Michaud en associant les artistes à des stars de cinéma, pointe la cruauté de leur destin : lorsqu'elles ne tournent pas, elles n'existent pas. D'où leur condamnation à espérer qu'un curator-réalisateur les contacte pour une exposition ou à devenir elles-mêmes les réalisateurs et acteurs de leur propre exposition. De fait, si cette mésaventure guette les artistes, l'exposition Nouvelles vagues, si elle place en priorité les curateurs et les met en lumière, laisse s'interroger sur l'issue d'un tel type de démarche. Faisant référence au film l'arroseur arrosé, Christophe Khim résume le piège du curator à travers le titre d'un article «l'accrocheur accroché ». En accordant au curateur les invitations habituellement adressées aux artistes que les curators permanents du Palais de Tokyo avaient la charge d'exposer, les curators peuvent craindre de connaître la même destination que les artistes, c'est-à-dire à devenir tributaires de personnages qui sélectionnent leur projet pour les retenir ou les écarter. Le rapprochement des expositions à la sphère 
du cinéma apparaît significatif dans le discours de médiation de l'institution d'une intention de représentation particulière à partir d'un modèle de pratique existant dont la pratique curatoriale devient en quelque sorte un ersatz. Aussi le travail de scénariste indépendant, qu'assure quelquefois le réalisateur de film, est-il associé à celui du curator, ce dernier «écrivant » dans l'espace de l'exposition un scenario comme le réalisateur en associant des plans filmés. La designer Matali Crasset, devenue présidente du FRAC Champagne Ardenne depuis novembre 2013, définit de la sorte la mise en espace des ouvres : « La scénographie est la mise en espace du scénario du commissaire. C'est un travail de valorisation des œuvres et d'accompagnement» (Crasset, 2011 : 129). Il faudrait alors travailler à une grille de valorisation pour mesurer ce que perdent et gagnent les œuvres d'art contemporain dans les expositions temporaires et thématiques afin de prendre garde, comme y appelle Jacinto Lageira, à ne « [...] pas déresponsabiliser le récepteur, car c'est lui qui fait exister l'œuvre en tant qu'objet d'art, mais aussi l'œuvre en tant que partage de sens " (Lageira, $2010: 85$ ).

L'expérience de l'exposition Nouvelles vagues au Palais de Tokyo a pu permettre de mesurer le poids de l'institution dans la mise en représentation de la pratique curatoriale. D'abord, parce qu'elle manifeste une invitation à exposer identique à celle qui pourrait s'adresser aux artistes. Ensuite, l'emploi de la métaphore cinématographique pour qualifier la pratique curatoriale, si elle est assurément un gage de reconnaissance pour les curateurs, n'en retentit pas moins comme un avertissement à considérer les déviances de l'industrie cinématographique quant au champ de l'art contemporain. En ce sens, le " thème " de l'exposition opère là encore à l'identique d'une exposition collective d'artistes contemporains. Au sens littéral, cette métaphore se vérifie : l'exposition est bien une mise en mouvement des images. Ce dernier point étant à considérer avec toutes les précautions nécessaires étant donné 1 . La tendance des œuvres à prendre la «forme » de dispositifs ou d'expériences qui ne sont pas des images à proprement parler. 2. Les qualificatifs employés pour désigner les pratiques de séquences, de séances, de plans, de scénarios, de story board, de progression, de programmation, de montage, de post production sont autant d'occurrences significatives pour montrer l'emprise désormais incontournable de ces acteurs dans la conduite du projet.

\section{BIBLIOGRAPHIE}

ARDENNE Paul (2013), « Packaging first. L'exposant plus que l'exposé », Archistorm, été, p.126-131.

BEYAERT-GESLIN Anne (2012), Sémiotique du design, Paris, PUF.

BOLTANSKI Luc, CHIAPELLO Eve (2011), Le nouvel esprit du capitalisme, réed., Paris, Gallimard. (Tel).

BOUTINET Jean-Pierre (1990), Antbropologie du projet, Paris, PUF. 
CRASSET Matali (2011), « Designer d'expositions/exposition de designers : Matali Crasset ». In Françoise Docquiert (dir.), Commissariat comparé. Histoires d'exposition, Paris, Paradox, p. 125-129.

DENI Michela (2009), "Lintervention sémiotique dans le projet : du concept à l'objet ». In Bernard Darras et Sarah Belkhamsa (dir.), Objets et communication, MEI, $\mathrm{n}^{\circ}$ 30-31, p. 87-98.

DRESSEN Anne (2009), «Le musée d'Art moderne de la Ville de Paris /ARC et le Palais de Tokyo : de quelques positions et collaborations inédites ». In Françoise Docquiert (dir.), Commissariat comparé. Histoires d'exposition, Paris, Paradox, p. 54.

FRONSACQ Julien (2009), «Le musée d'Art moderne de la Ville de Paris/ARC et le Palais de Tokyo : de quelques positions et collaborations inédites ». In Françoise Docquiert, Commissariat comparé. Histoires d'expositions, Paris, Paradox, p. 60.

GLICENSTEIN Jérôme (2009), L'art : une histoire d'expositions, Paris, PUF (« Lignes d'art »).

GRENIER Catherine (2013), La fin des musées? Paris, Éd. du Regard.

JOUVENET Morgan (2001), « Le style du commissaire. Aperçus sur la construction des expositions d'art contemporain ", Sociétés Ė Représentations 1, n 11 , p. 325-348.

LAGEIRA Jacinto (2010), La déréalisation du monde, Réalité et fiction en conflit, Nîmes, Jacqueline Chambon.

LIPOVETSKY Gilles, SERROY Jean (2013), L'esthétisation du monde vivre à l'âge du capitalisme artiste, Paris, Gallimard.

LOISY Jean de (2013), Éditorial. In Guide de l'exposition "Nouvelles vagues ", Paris, Palais de Tokyo. [non paginé].

MANGION Éric (2001), "L'effet bande-annonce ». In Catherine Millet (dir.), Écosystèmes du monde de l'art, pratiques marchés institutions, mondialisation, Art press, $n^{\circ} 22$ spécial, p. 35-37.

MARTEL Frédéric (2010), Mainstream. Enquête sur la guerre globale de la culture et des médias, Paris, Flammarion.

MENGER Jean-Michel (2005), Profession artiste. Extension du domaine de la création, Paris, Textuel.

MICHAUD Yves (1989a), L'artiste et les commissaires. Quatre essais non pas sur l'art contemporain mais sur ceux qui s'en occupent, Nîmes, Jacqueline Chambon.

MICHAUD Yves (1989b), «Éditorial », Les cabiers du MNAM, n² 29, automne.

MORIN Edgar (2008), L'esprit du temps, Paris, Armand Colin.

PUGNET Natacha, dir. (2011), Jeu(x) d'exposition. Pratique et théorie de l'exposition, Nîmes, École supérieure des Beaux-Arts, (coll. Hôtel-Rivet), p. 122-135.

SARTRE Jean-Paul (1980), Plaidoyer pour les intellectuels, Paris, Gallimard, 1980.

SCRIVE Martine (1994), «Qui fait une exposition ? Du commissaire à l'équipe projet », Publics et Musées, vol. 6, n 6, p. 99-104.

VANDER GUCHT Daniel (1998), L'art contemporain au miroir du musée, Bruxelles, Éd. La lettre volée. (Les essais)

VIAL Stéphane (2010), Court traité du design, Paris, PUF. 
Résumé : En orchestrant des navigations possibles à travers des données en perpétuelle augmentation, le phénomène de la curation conduit à s'interroger sur une mise en tension entre projet et design. L'article propose une étude de l'exposition d'art contemporain temporaire Nouvelles vagues qui réunissait une vingtaine de projets curatoriaux au Palais de Tokyo. L'objectif de cette analyse vise à proposer une approche de la représentation de cette pratique du projet qui fédère des acteurs, des lieux, des intentions - qui les manifeste par l'institution culturelle et dont le design permet de prendre la mesure.

Mots-clés : curation, musée, exposition.

Abstract: By orchestrating possible ways through ever-increasing data base, the phenomenon of curating raises problematics questions between project design. This paper presents a study of curatorial practice focused on temporary contemporary art exhibition Nouvelles vagues which is a display of twenty curatorial projects at the Palais de Tokyo. The aim of this analysis is to propose an approach to curating as a practical project which brings together actors, locations, intentions - that manifest it and whose design allows to understand the full extend of.

Keywords: curating, art center, exhibition, display. 
